\title{
Lightning Protection System to the Indian Satellite Launch Pad
}

\author{
GR Nagabushana, Joy Thomas, Udaya Kumar
}

D Venkateshwara Rao, PV Panduranga Rao
Introduction: Any launch mission forms a complex $\&$ expensive process. Intensive care and precautions arc to be taken for a safe and successful launch. Also, the satellite launch system forms a tall structure standing on a plane terrain. As a result, a lightning strike rate to it becomes more probable. Therefore, extensive care needs to be taken in shielding the launch system against natural lightning. Lightning protection systems built with differing principles have been in use at different launch centers of the world. This paper describes the lightning protection system that has been installed at the Indian satellite launch center -SHAR,

The launch system at SHAR, consists essentially of a Mobile Service Tower (MST) with an Umbilical Tower (UT). MST is a steel structure containing assembly equipment and all the accessories. UT (气 51) supports the necessary interconnections between the controllfeedlcheck-out systems and the launch vehicle (LV). During the assembly, the MST will be housing the whole setup $\&$ it will be relatively safe for the launch vehicle and the satellite. However, prior to the launch, MST will be retracted exposing both UT and the vehicle to lightning environment. In this situation, the control and data cables running between UT and the vehicle $\&$ satellite are very much prone to direct and indirect electromagnetic couplings from lightning. There are a few buildings in the vicinity of the launch site housing the sensitive control \& measuring equipment and also the rocket fuel processing \& storing units. Former is sensitive to any electromagnetic activity in the MST/UT/Vehicle and latter for the heating/arcing resulting in possible ignition in any section of the fuel transport systems. The location being next to Bay-ofBengal is known for frequent cyclones \& thunderstorms activities. Therefore, a reliable protection system is necessary for protecting the launch mission from the natural lightning activities. In the following section, details of the development are described.

Protection svstem: Lightning protection systems may be thought of as comprising:

(a) a Receptor or Air termination, (b) Transmitter \& (c) Ground dissipator. The receptor is the tip of a tall standing structure which is intended to intercept the lightning path which otherwise could have landed on system to be protected. The transmitter in its simple form is the tower that conducts the lightning current to the ground. However, due to large magnitude and rate of rise of the lightning currents, a very high voltage could he generated in the transmitting system leading to secondary flashovers/damages to the LV. Therefore, it has to be designed such that the voltages generated are within the permissible limits and any induced voltages in sensitive circuitry are within acceptable limits. The grounding system needs to dissipate efficiently the current of the lightning stroke intercepted by the protection system safely into the ground. It has to ensure safety to the personnel at the site and any coupling with data and power cables by electromagnetic inductions should be tolerable. In the following sections, design principles employed are discussed.

The main objective of the intercepting system is to establish an electrical contact with the descending stepped leader thus protecting the object. As a consequence of this requirement, the interceptinglair termination system needs to stand taller than the system to be protected. The protection system has to be at a reasonable distance away from the UT \& LV, which demands a distributed configuration encompassing UTIMST. Noting that only the air termination/receptor system needs to be distributed to encompass the protected system, for the SHAR lightning protection system, a three tower system was configured making an equilateral triangle with UT \& LV approximately at its center and conductors running between them at their top. On the top of each tower, there is a mast of height $10 \mathrm{~m}$, put as the first seeker of the descending downward stepped leader. These masts are fitted with small cross-arms for increasing their effectiveness. The towers with the inter linking conductors are intended to form a broad area receptor. These conductors are expected to receive flashes that are missed by the tower topslmasts. With a strike to any one of these elements, ground coimections is achieved.

For deducing the height of these towers, the classical rolling sphere model is employed [1]. The radius of the rolling sphere (the Striking distance [21) $R_{s}$ has been related to the peak current (expressed in $\mathrm{kA}$ ) in the stroke through: 


$$
R_{s}=k \bullet I_{0}^{0.65} \quad \mathbf{m}
$$

The values for $\boldsymbol{k}$ have been given in the range 6.7 to 10. The latter value gives a more conservative estimate from protection point of view. Now, a sphere with radius of $R_{s}$ is rolled over the receptor system and the height for the towers is decided which will be sufficient for intercepting currents in excess of $I_{c:}\left(I_{c}\right.$ is the critical current above which the protection scheme is fully valid). If the stroke occurs to the mid span/tower, the potential generated needs to be calculated in order to ensure that the potentials will not rise to a value to cause a secondary flash from the receptor to the UT/LV. For this purpose, the whole protection system is modeled by a transmission line network. For worst possible current magnitudes and rise times, computation of the voltages at the various locations that are nearest to the UT/LV are to be made and the available clearances verified. During a stroke to the mid-span, only possible way for minimizing the potential rise is to decrease the surge impedance. This can only be effected by increasing the number of conductors. However, this increase will add on to the total cost of the conductor and the tower (as it has to take heavier cantilever load).

Regarding the ground dissipator, extensive ground conductors will be necessary for distributing the lightning current safely into the earth. At the tower foot, the tower base with the driven rod is expected to form an excellent ground connection. In order to minimize the step potentials and the interference with the data/control lines a circular counterpoise linking all tower bases is adopted. In order to counter the high soil resistivity during dry seasons, driven rods are used at regular intervals along this circular counterpoise.

From the cost considerations it is evident that the total cost of the system increases with the height of the tower \& number of conductors at the top. Design for withstanding cyclonic storms will further add to the overall cost.

Details of the protection system:- After considering all the above requirements \& constraints the following design has been arrived at (Figure 1).

Number of towers: 3 Located at the vertices of an equilateral triangle of base $180 \mathrm{~m}$.

Height of the towers: $120 \mathrm{~m}$

Tower base dimensions: $14 \mathrm{~m} \times 14 \mathrm{~m}$

Size of the conductor: $1.2 \mathrm{~cm}$

Number of conductors: 6

Details of the counterpoises:

Radios of the 2 concentric ring counterpoise: $115 \mathrm{~m} \& 165 \mathrm{~m}$.

Depth of burrying: I5m.
Interval between driven rods: GI pipes at every $20 \mathrm{~m}$; Penetration depth: $13 \mathrm{~m}$.

\section{Evaluation of the system:}

(a) Effectiveness of the protection: The conductor swing due to wind was also considered in arriving at the tower height. The final design was found to allow strokes with current less than $15 \mathrm{kA}$ can sneak through the protection system towards the UT/LV (only if it arrives absolutely vertically along the axis of UT/LV). It may be noted that the design principle itself allows strokes with current less than a critical value $I$, to land on protected system.

(b) Strokes to protection system: A near-worst case analysis with a stroke current of $200 \mathrm{kA}$ was considered. Whole protection system was represented by transmission line equivalents. Towers were replaced by lines having a surge impedance of $125 \Omega$ [3] and a length equal to tower height. The conductors at the top of towers are also replaced by lines with a surge impedance of 2100 calculated from:

$Z o=42.39 \ln (G M D / G M R)$. Where GMD \& GMR are the geometric mean distance and geometric mean radius respectively. For the ground connection a constant ground resistance of $150 \Omega, 5 \Omega$ and a transient resistance decreasing from $150 \Omega$ to $5 \Omega$ were considered. Latter was employed to model the transient behavior of ground rods/counterpoises.

The computed potentials during strokes to mid span and the tower top are shown in figure $2 \& 3$ for some typical cases. The computed voltages indicate a worst case maximum value of $23 \mathrm{MV}$ [4]. The minimum clearance between the lower most conductor after considering the sag and the top most point on LV is about $54 \mathrm{~m}$, which is believed to be sufficient to withstand the computed maximum potential difference. The maximum voltage gradients and voltages on the ground are also computed and for a better personal safety a fence will surround the tower base.

An experimental evaluation was also carried out on scaled models of the protection system in the laboratory with $4 \mathrm{MV}$ impulse voltage generator. The experiments with their limitations have proved the effectiveness of the protection system developed for the SHAR.

Conclusions: The requirements of a lightning protection system to a satellite launch site are discussed. The major steps followed in evolving a suitable protection system for the SHAR-the Indian Satellite launch site, are presented. 1he final design 
has been verified with the tolling sphere model for the effectiveness of the protection. A post stroke analysis with the transmission line representation of the protection system has also verified the design against any back flashovers. Experimental verification was also carried out with some scaled models at the laboratory.

During the previous lightning season, it is believed that some strokes did occur to the system without causing any untoward incident.

\section{References:}

1. T Horvath, Computation of Lightning Protection, Research studies press ltd., John Wiely \& Sons Inc., 1991.

2. K. Berger, The Earfh Flush, in book Lightning, Part1-Physics of Lightning, Edited by R.H. Golde. Academic Press. 1977.

3. T Yamada, A Mochizuki, J Sawada, E Zaima, T Kawamura, A Amctani, M Ishii \& S Kato, "Exoerimenlal Evaluation of a UHV Tower Model for Lightning Surge Analysis", IEEE Transaction on Power Delivery, Vol. 10, No. 1, January 1995.

4. GR Nagabushana \& Joy Thomas, Final report on "Development of a Lightning Protection Scheme for Launch Pad at SHAR", Department of High Voltage Engineering, IISc.

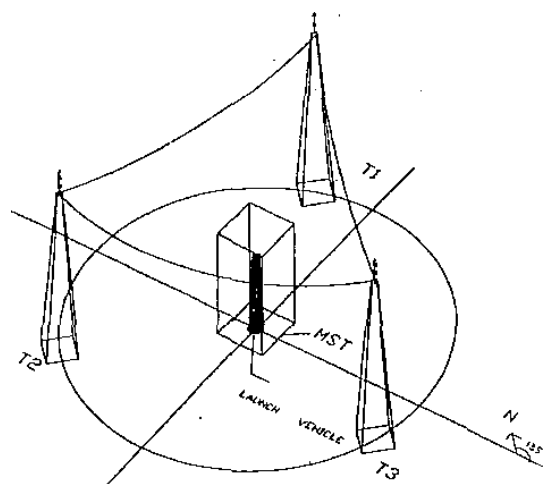

Figure 1. Schematic of the protection system on site

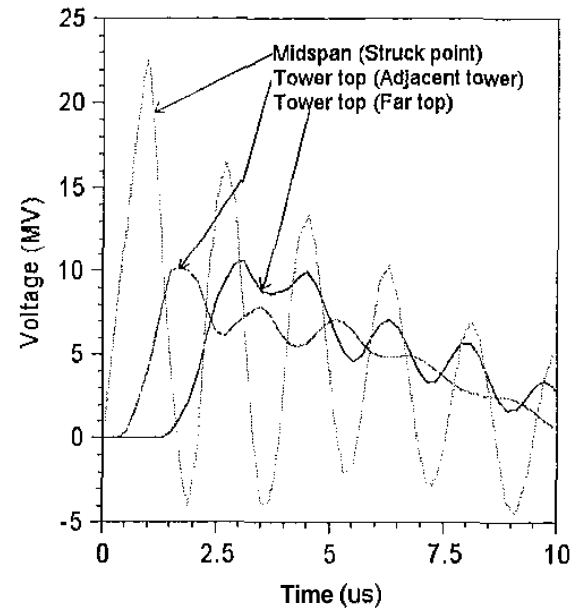

Figure2. Induced voltages during stroke to midspan 


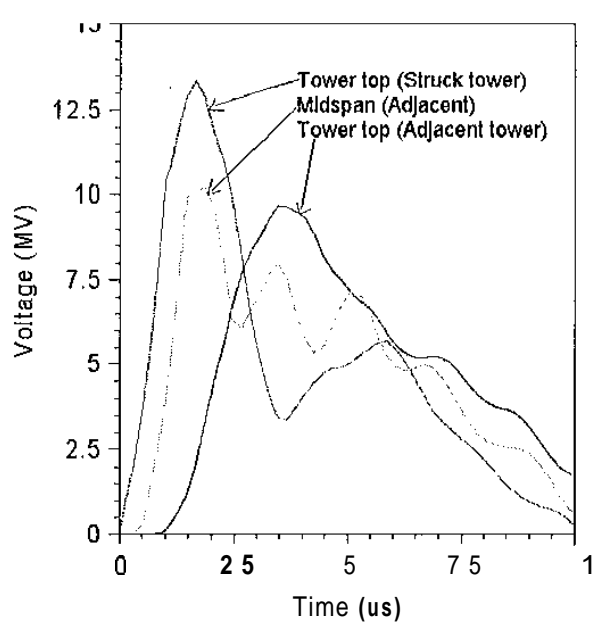

Figure 3. Induced voltages during stroke to tower top

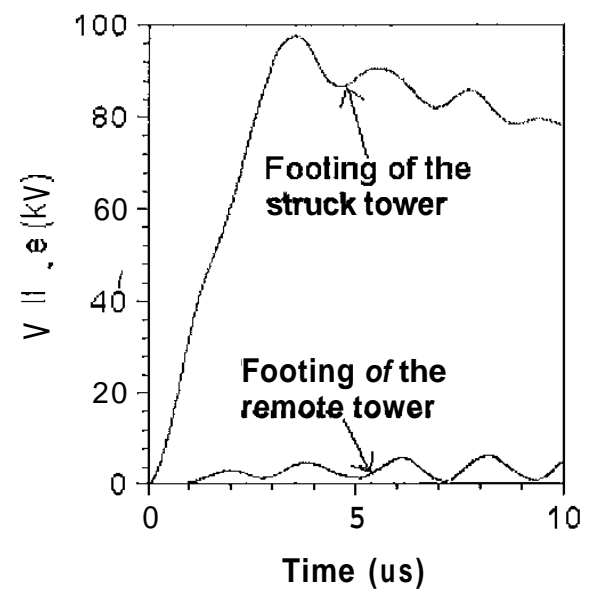

Figure 4. Voltage at tower footings (Stroke to tower top)
Address of the Corresponding Author: Prof. G.R. Nagabhushana,

Department of High Voltage Engineering, Indian Institute of Scicncc, Malleswaram, Bangalore 560 012. India.

Email:-grn@hve.iisc.ernet.in

Phone:- +9l-080- 3092376,3092372

Pax:- $+91-080$ - 3341683, 91- 080 - 3342085 . 